\title{
Light trapping in translucent samples and its effect on the hemispherical transmittance obtained by an integrating sphere
}

\author{
Jacob C. Jonsson ${ }^{a}$ and Arne Roos ${ }^{a}$ and Geoff B. Smith ${ }^{b}$ \\ ${ }^{a}$ Dept. Materials Science, Uppsala University, PO Box 534, SE-751 21 Uppsala, Sweden \\ ${ }^{b}$ Dept. Applied Physics, University of Technology Sydney, PO Box 124, Brodway NSW 2008,
} Australia

\begin{abstract}
When a beam of light is incident on a translucent sample, a significant fraction of the light is scattered at high angles. Some of this scattered light may be trapped inside the substrate through multiple reflections and total internal reflection, similar to light coupling into an optical fiber. The trapping depends on factors such as the surface roughness of the external surfaces and/or the size and distribution of scattering particles inside the sample. The scattered light may thus escape out of the sample at a shifted position relative to the incident beam. This leads to port losses in an integrating sphere. The detected signal from the light entering the sphere then underestimates the hemispherical transmittance. In this paper the signal versus lateral position has been measured in an attempt to estimate the error and to find an extrapolation procedure for the correct transmittance value. The lateral measurements were carried out by moving a detector behind the sample, a procedure carried out at several angles of incidence. Different illumination methods have also been studied both theoretically and experimentally to further investigate what effect light trapping can have when characterising scattering samples.
\end{abstract}

Keywords: Integrating spheres, light scattering, optical trapping, hemispherical transmittance

\section{INTRODUCTION}

Integrating spheres ${ }^{1}$ are commonly used for characterising the total and diffuse transmittance of different samples. It is still impossible to manufacture an ideal sphere, since design decisions result in different systematic errors. There have been several studies of these effects and how to compensate for them. Different port effects have been studied, e.g. where asymmetric samples scatters light to give an incorrect value ${ }^{2}$ or where the sample is not close enough to the sphere. ${ }^{3}$ The sphere response is also expected to be different for specular and diffuse samples. ${ }^{4}$ Since most samples have both a diffuse and specular component this requires a good knowledge of the relation between the two components. Port-losses can also be compensated for systematically. ${ }^{5}$ All of these studies are focused on what happens when light enters the sphere, and they generally neglect the fact that a scattering sample, under certain conditions, can divert light so that it never enters the sphere.

The most important aspect of this paper is the fact that light is laterally transported, or side-shifted, as it travels through a scattering sample. Side-shift is not the same as scattering; side-shift is a measure of where on the sample light exits with respect to where it entered rather than in what angle it exits. Even though the term side-shift might so suggest, there is no requirement for image preservation when it is used. Side-shifted light might smear, re-focus or arbitrarily distort the incident image.

The side-shift effect can cause transmitted light to exit the sample in such a fashion that it is not collected by the sphere. This side-shift is dependent on from where the scattering originates in the sample; front surface, back surface, the bulk, or a combination thereof. The result of side-shift in a scattering sample is usually an enlargement of the exiting light spot compared to the illuminating light spot. Hence it is also important to study how this side-shift behaves for different illumination situations.

Further author information: (Send correspondence to J.C.J.)

J.C.J.: E-mail: jacob.jonsson@angstrom.uu.se, Telephone: +46 184717234 

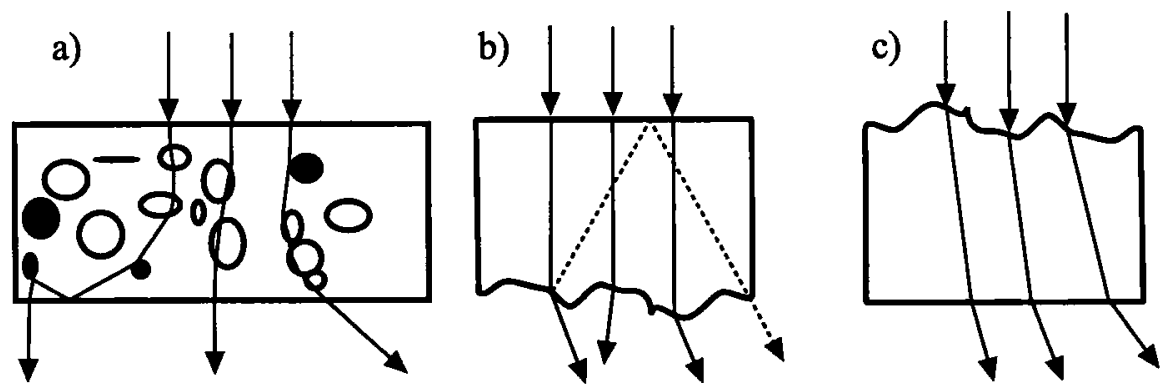

Figure 1. Three different cases of scattering. In a) a bulk scattering material may both have reflective and diffracting scattering centers in it. Case b) and c) emphasize the difference in sideways shift in the exiting light depending on if the light enters or exits through the scattering surface.

\section{THEORY}

\subsection{Material}

Materials that scatter light can do this in several different ways. Figure 1 shows three different situations that is studied in this paper. Each situation results in a different approach on how to characterise how light is transported sideways inside the sample.

For bulk scattering materials (Fig. 1a)), the side shift has to be simulated with ray tracing or some other method. Depending on if the material has diffracting and/or reflecting scattering centers the ray will travel differently. Both cases shown in Fig. 1a) and Fig. 1c) can trap light with total internal reflection if it reaches the exit surface at a high enough angle.

The case in Fig. 1b) shows that any shift comes from internally reflected rays, i.e. the only light that is shifted sideways before leaving the sample has been internally reflected at least twice.

There are of course samples which combine bulk scattering with surface scattering, and for such samples the effects have to be combinded to find the correct light distribution.

\subsection{Sample Illumination}

The illumination of the sample can contribute to the side-loss effect. We have studied three different cases, point-like illumination in the center of the port, wide-area illumination where the light spot is much larger than the port, and finally, the Labsphere $150 \mathrm{~mm}$ integrating sphere accessory to the Perkin Elmer Lambda 900 spectrophotometer serves as an example of an arbitrary shaped light spot.

In all cases the incident light is considered to be parallel, and homogeneous in intensity across the illuminated area. However, a note on how a varying intensity can be incorporated is included in the third case.

\subsubsection{Point-like illumination}

In this case we simply treat the light as a point at the center of the sphere port. Results from this section will be used in the following two cases.

All light is assumed to hit at one spot of the sample. This spot is small enough to be treated as one coordinate with respect to the entrance port, but large enough to allow for scattering in all directions. This is an important assumption since in principle one could claim that a single ray would always propagate along the same path and not create a scattering pattern.

In Fig. 1b) the only side-shifted contribution comes from light that has been reflected from the scattering back surface, reflected in the front surface and finally transmitted through the scattering surface. This contribution is often negligible for low-reflecting materials such as glass (where $99.8 \%$ of the transmitted light is not internally reflected), but rapidly becomes an issue for samples with high reflectance of both the front and the back surface. 
It is also worth considering that reflectance is angle-dependent and a rough surface will have a locally varying reflectance due to the structure. Light scattered inside the sample that exit at oblique angles of incidence might be totally internally reflected even though the material has a low reflectance at normal angle of incidence.

One way to measure the side-shift is to measure the scattering angle distribution and from this calculate the internal scattering distribution simply by using Snell's law. The internal scattering angle in combination with sample thickness is then used to determine the mean-deviation for each refiection to get the resulting side-shift.

Situations shown in Fig. 1c) will generally result in a distorted image, generally enlarged, of exiting light compared to the incident light spot due to scattering induced side-shift. Exceptions from this could occur in in homogeneously scattering samples. In the case with low reflecting surfaces, it is again possible to neglect side-shift from internally reflected light. The side-shift of the primary transmittance is calculated using the same internal scattering angle distribution as in the previous case. If the sample surfaces are highly reflecting the internal reflections must be considered.

For bulk scattering samples with multiple scattering centra, as shown in Fig. 1a), it is generally hard to find a simple model that handles the scattering. It is then suggested that ray-tracing models are used to simulate both scattering and side-shift.

\subsubsection{Wide area illumination}

The use of wide area illumination for integrating spheres have been described by and investigated by e.g. Platzer ${ }^{6,7}$ and Milburn. ${ }^{8}$ This allows for measuring thick, inhomogeneous, bulk-scattering samples at near normal as well as at oblique angles of incidence. For such samples point-like illumination measurements can cause major errors if incident light is scattered and/or side-shifted so that no light enters the sphere. By illuminating a larger area than the port it is assumed that the sample will scatter the correct amount into the sphere.

By using the theory described for point-like illumination in the previous section, the wide area illumination is divided into several point-like sources. Each point is assigned coordinates relative to an assigned center of the sample; it is convenient to select the center to match the center of the sphere port. Then it is possible to handle each point as described above to find each individual contribution which are then added together to find the total contribution.

For calculations on such samples it is important to understand that the total intensity illuminating the sample is larger than what is detected with the reference measurement. The reference should be the port area divided by the wide-beam area for a beam with homogeneous intensity distribution over the complete beam. For illumination situations where light intensity varies over the beam spot it is necessary to compensate for this by, e.g. incorporating a weight factor into each point-like source.

\subsubsection{Arbitrary shaped illumination}

The Lambda $900150 \mathrm{~mm}$ integrating sphere used in this study has a complex illumination spot as shown in Fig. 2. This case is handled by dividing the beam into spot-like parts just as in the wide area illumination case. A method to determine the spot coordinates is taking a digital photo of the light spot and assume that each pixel in the beam image is a spot-like source. The resolution of the image will define the number of individual sources, increasing both calculation times and accuracy with increasing resolution. Distances in pixel space can easily be translated to distances in lateral units using the size of the sphere port clearly seen in the image.

\subsection{Calculation of port acceptance distribution}

The amount of light that enters the sphere depends on three things: the shape and size of the incident beam, the shape and size of the port, and the side-shift generated by the sample. By dividing the incident beam into point-like beams it is possible to take each part of the beam and use the sample side-shift to calculate how light is smeared over the port. With that information it is straight forward to determine how much light that entered through the port for that given part of the incident beam. Integration of all parts of the beam will then result in a single value of how much of the transmitted light that entered through the port.

The illumination of the entry port of the Lambda $900150 \mathrm{~mm}$ integrating sphere is shown in Fig. 2. The port is conveniently circular which simplifies calculations for symmetry reasons. The calculations can be done for a square port as well, albeit it is a bit more complex. 


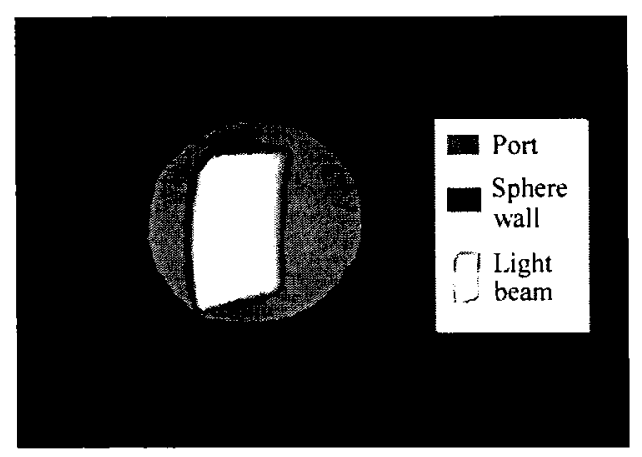

Figure 2. Digitally enhanced photograph of the light spot at the entrance port of a Lambda $900150 \mathrm{~mm}$ integrating sphere.

Figure 3 shows the geometry for a point-like incident beam at a distance of $d$ from the center of a circular port with radius $R$. Light is side-shifted a distance $r$ in a random direction while traveling through the sample. Assuming a homogeneous sample this randomness results in equal probability for all parts of the dashed circle. Then the probability that a ray will enter the port is equal to the ratio between the length of arc inside the port and the the length of the perimeter $(=2 \pi r)$. This ratio is simply given by $2 \theta / 2 \pi$, and $\theta$ is easily calculated from the law of cosines

$$
\theta=\arccos \left(\begin{array}{c}
r^{2}+d^{2}-R^{2} \\
2 r d
\end{array}\right)
$$

resulting in the final probability

$$
P(d, r)=\frac{1}{\pi} \arccos \left(\begin{array}{c}
r^{2}+d^{2}-R^{2} \\
2 r d
\end{array}\right) .
$$

This equation, naturally, only holds when the triangle inequality holds $(r+d \geq R)$. It is worth noting that in the limit of $r+d \rightarrow R^{+}$then the function $P(r, d) \rightarrow 1$ (if $r+d \leq R$ we have $\mathrm{P}=1$ ). One can also see that the incident beam can be outside the port without violating Eq. 1 . This is an important result for using it in wide-area illumination comparison.

Once the probability function is obtained the matter of integrating over all parts of the incident beam, as well as over all $r$ remains in order to find the amount of transmitted light that enters the port. This value must be divided by the amount of light that enters the port in a reference measurement, which is represented by $P(d, 0)$

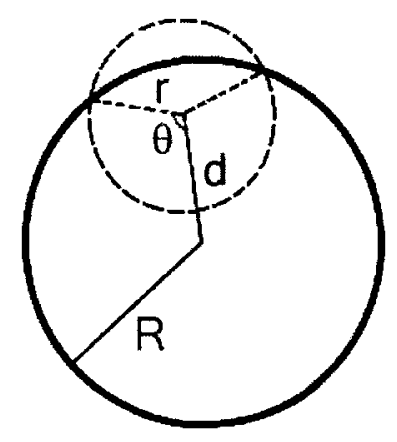

Figure 3. The geometry of a point-like light beam that strikes the sample at a distance $d$ from the center of the circular port with radius $R$. The beam is then side-shifted the distance $r$ in an arbitrary direction. 
since there is a constant side-shift of zero in the reference measurement. The correction factor used to find the true transmittance from the measured transmittance is then given by

$$
T_{a}=\begin{gathered}
\int_{d} \int_{r} I(r) P(d, r) d r d d \\
\int_{d} P(d, 0) d d
\end{gathered},
$$

where $I(r)$ is the normalised side-shift distribution of the sample. To find the true transmittance the measured transmittance should be divided by $T_{a} . T_{a}$ can only be larger than one for wide area illumination cases, and is expected to be exactly one for perfectly specular samples (since $r=0$ for such samples).

\section{EXPERIMENT}

A $4 \mathrm{~mm}$ solar collector structured cover glass, GERRISOL Weiss no. 502, was chosen as a demonstrating experiment. One side of the sample has a large-scale scattering structure, the other side is smooth.

The sample was illuminated by a $5 \mathrm{~mm}$ collimated beam at a wavelength of $550 \mathrm{~nm}$. To study where light exited from the sample, a silicon detector was masked so that only a 1.2 by $1.2 \mathrm{~mm}$ square was active in the center of it. The masking resulted in sufficient spatial resolution for tracking where the light exits the sample.

The sample was oriented both with the structured side facing the light and away from the light. For each orientation the measurement was carried out at three different angles of incidence. First at normal and then at $30^{\circ}$ and $45^{\circ}$. All orientations are shown in Fig. 4.

The detector was positioned as close to the sample as possible, less than $0.5 \mathrm{~mm}$, and then moved along a rail relative to the illuminated position of the sample. The position along the rail was controlled by a screw which moved the detector $1.2 \mathrm{~mm}$ per full turn. Both sample and reference measurement was carried out for each individual detector position. There are two reasons for this, firstly the instrument is single-beam and secondly to avoid errors caused by the limited repeatability of the detector movement.

The zero position of the detector was defined with respect to the normal angle to where the light spot hit the structured surface, as shown in Fig. 4. With this definition the specular maximum for measurements at oblique angles of incidence being detected away from the zero position.
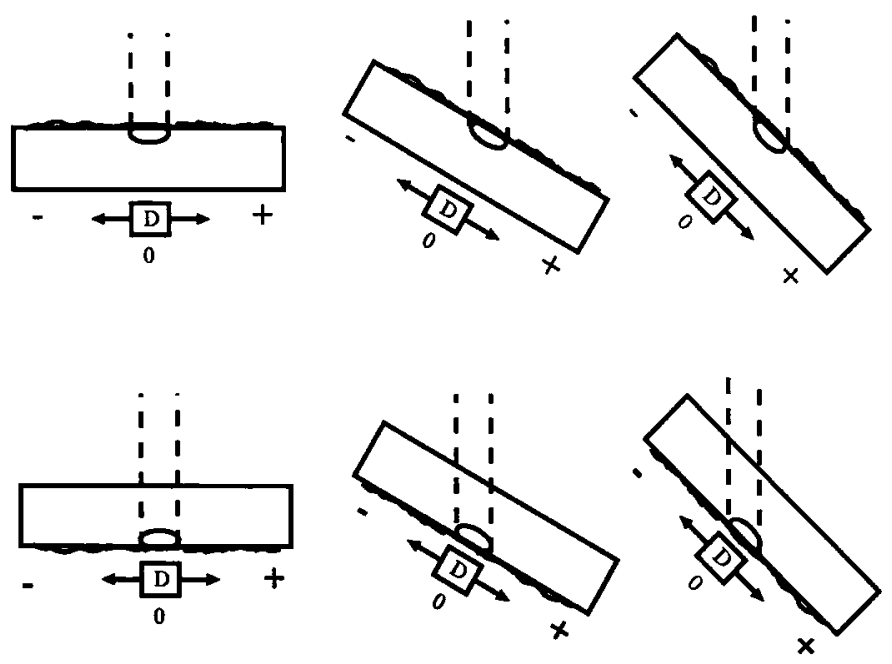

Figure 4. The six different sample orientations used. The light is incident from the top of the picture in each individual case. The detector zero position for each orientation is also defined. 


\section{RESULTS}

\subsection{Experiments}

Samples, such as the studied structured cover glass, exhibit some side-shift at normal angle of incidence, which is increased in an asymmetric fashion at oblique angles of incidence. As shown in Fig. 5, the broadening is more prominent in the case where the scattered light has a longer distance to travel within the sample. That effect is seen when the structured surface is facing the incident beam. As the angle of incidence increases, so does the distance light travels through the sample, hence an increased side-shift can be detected.

Peak broadening is dependent on angle of incidence since the path the detector moves changes with angle of incidence. Hence, the detector must be moved a distance $x$ to cover the full width $d$ of the beam spot, where

$$
x=\begin{gathered}
d \\
\cos \theta
\end{gathered}
$$

for an angle of incidence $\theta$. But since the width of the beam spot is unchanged, this corresponds to an increase in spatial resolution rather than an actual side-shift of the incident light. This can be observed in the reference measurements for oblique angles of incidence. Multiplication by the factor $\cos (\theta)$ makes it is possible to get side-shift with respect to the incident beam width rather than the size of the beam projected onto the sample.

Measuring the side-shift in the described fashion does not collect all light that is side-shifted the given distance since data is only acquired along on line (or rather a ribbon) along the sample, rather than all over the sample. Hence, the proper side-shift value is aquired by compensating for the corresponding circumference for each given $\mathrm{x}$-value.

\subsection{Port acceptance calculations}

As described in Sect. 2 it is possible to use the measured side-shift data in combination with the light spot shape to calculate a correction factor for how much light missed the entrance port due to side-shift.

The digital image shown in Fig. 2 was analysed to find the light spot distribution. The distance between each light spot pixel (approximately 22000) and the port center was determined and the result binned to a
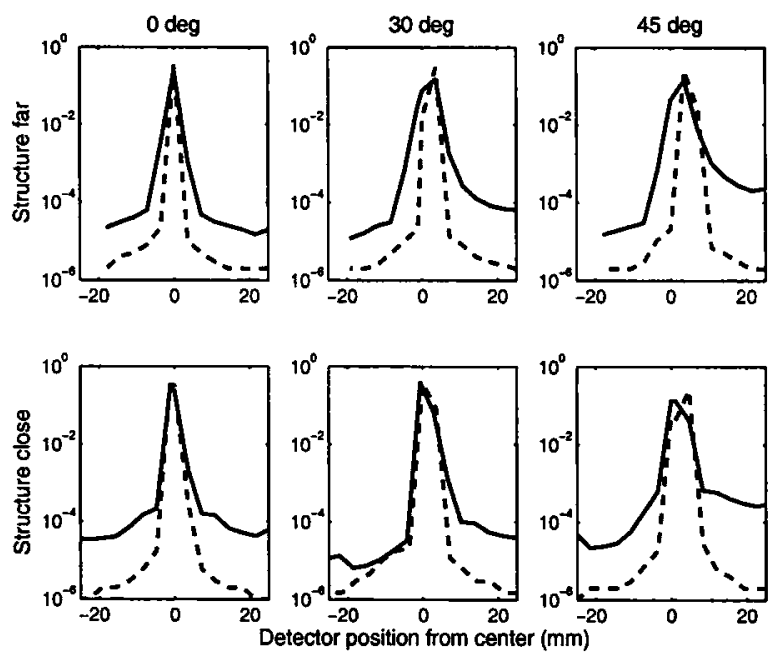

Figure 5. Results from six measurements corresponding to the orientations given in Fig. 4. The upper row is for measurements with the structured side of the sample away from the detector and the lower row contains results from measurements with the structured side towards the detector. 

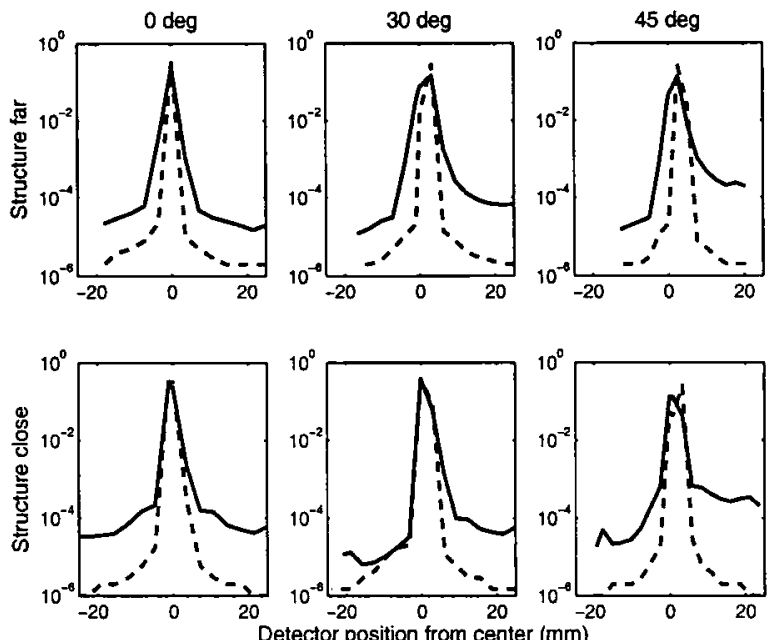

Figure 6. As Fig. 5, but with a modified $x$-axis corresponding to the normal angle of incidence plane in all cases rather than to the actual angle of incidence. This gives an $x$-axis with respect to the incident beam rather than the illuminated part of the sample.

distance distribution with a resolution of $0.1 \mathrm{~mm}$, as shown in Fig. 7. The binning is convenient for avoiding unnecessary calculations since each distance, regardless off position, will result in the same loss due to port and sample symmetry.

The amount of light entering the sphere can now be calculated with Eq. 3, using the side-shift measurements as a range of values for $r$ and the binned data from the photograph as a range of values for $d$. Integrating over both ranges gives a single value for the relative fraction of light entering the port.

The value for the case with the structure close to the port was calculated to 0.9974 , and the value for the case with the structure away from the port was 0.9935 . The total transmittance as measured and corrected are shown in Fig. 8.

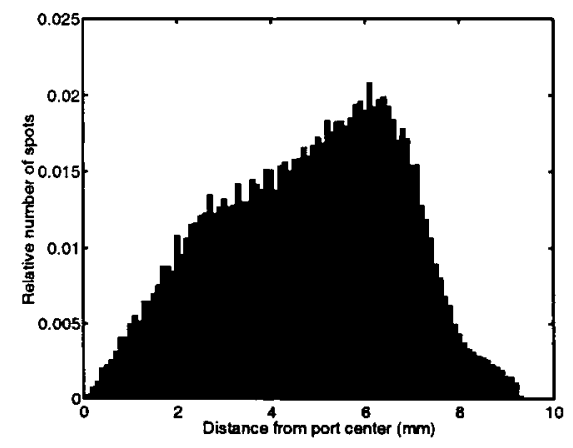

Figure 7. Binned distribution of the distances to the port centre of point-like beam spots at the Lambda 900 entry port (as seen in Fig. 2). The number of elements in each bin has been scaled to the total number of elements so that the sum of all bins equals one. 


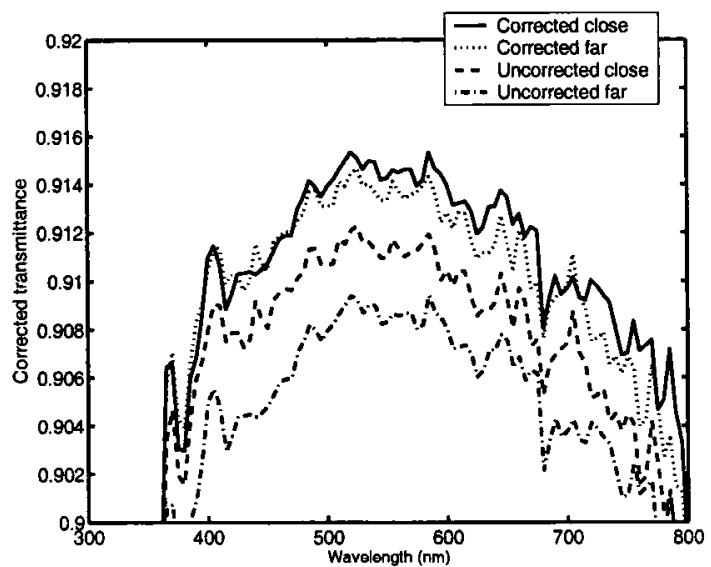

Figure 8. Experimental and corrected values for the total transmittance depending on sample orientation. The correction results in a significantly smaller difference between the two orientation.

Figure 9 shows the absorption of the samples which, of course, should be the same independent of orientation. This is the case for the absorption calculated using the corrected values. The actual value of the absorption is also more resonable since the sample studied is a low-iron glass.

The reflectance measurements have not been corrected for several reasons. Side-shift measurement of the reflected light is complicated since the detector would shade the sample from the incident light. The Lambda 900 instrument also features a different light spot shape at the reflectance port. Even if one chose to use the same side-shift data and finding the correct distance distribution, the correction is only of the order if instrument noice, making it negligible.

In an attempt to reduce the side-shift induced port loss, a focusing lens was inserted in front of the entrance port. This resulted in a smaller image which was centered on the port, increasing the minimum distance needed

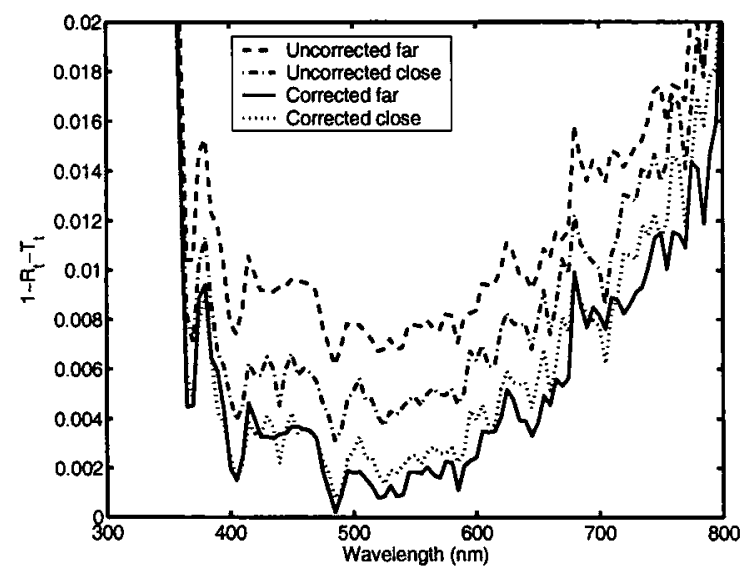

Figure 9. Experimental and corrected values for the absorption. The total transmittance depending on sample orientation. The correction results in a significantly smaller difference between the two orientation. 


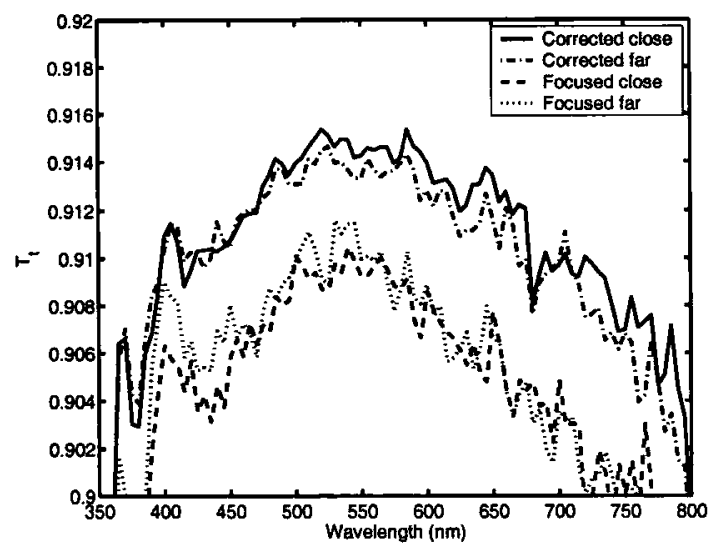

Figure 10. Corrected values for standard measurement and a measurement with a more focused image on the entrance port. Both methods give the same transmittance for both orientations but the level is lower for the focused measurement.

to transport light away from the port. In this case the calculated loss factors were 0.9997 and 0.9998 for the cases with the structure away from the port and towards the port, respectively. The corrected focused measurement and the corrected standard measurement are shown in Fig. 10.

The measurements with the focusing lens resulted in a lower transmittance even though it was the same for both orientations. Care was taken to tilt the lens so that multiple reflections between the sample and the lens should not enter the sphere. The focusing results in a slightly higher angle of incidence which could give rise to a higher reflectance, but for reasonably unpolarized light this increase in reflectance is less than 0.0001 for the low angle change that occurred in the experiment. However, focusing the light on the entrance port creates a distortion of the image at the reflectance port where the specular light is first reflected inside the sphere. The edge of the reflectance port is not as reflecting as the sphere wall which results in a too low detected signal. The low-scattering character of the studied sample made it sensitive to this port-edge effect. In addition, the assumption that the sample surface is homogeneous is not true when using a focused incident light beam. This fact, in combination with the port edge loss effect, made it possible to acquire a measured value for the total transmittance which varied by approximately $\pm 0.3 \%$ depending on which part of the sample that was measured upon. This is the most probable explanation to the descrepancy shown in Fig. 10 between the two pairs of transmittance curves.

There was no possibility to perform wide area illumination experiments with the used integrating sphere. Calculation of port acceptance factors were carried out for different illuminating beam radiuses assuming a homogeneous intensity distribution across the incident beam spot. The correction factors for the case with the structure closest to the light source ranged from 0.9494 to 0.9143 as the radius of the incident beam was decreased from $100 \mathrm{~mm}$ to $10 \mathrm{~mm}$ (same as the port size). The opposite orientation led to a range from 0.9701 to 0.9470 for the same spot sizes. This indicates that wide area illumination is a situation where this kind of correction is more important, even for samples with little side-shift.

\section{CONCLUSIONS}

It is hard to determine a general correction factor for side-shifted light, but there are straight-forward ways to compensate for it. The procedure to experimentally determine the side-shift makes it possible to make corrections for port losses when small sized scattering samples are investigated with standard integrating spheres.

The samples examined in this study have a small side-shift effect but even that could be compensated for properly using the suggested methods, removing an unphysical apparent additional absorption loss as shown in Fig. 9. 
Proper instrument design can avoid many of the problems with side-shift in scattering samples. However, more often than not users are not able to design their own instruments and must use whatever is available, hence there is a need for proper corrections.

\section{ACKNOWLEDGMENTS}

The work was carried out with financial support from the Swedish Strategic Science Foundation (SSF).

\section{REFERENCES}

1. R. Ulbricht, "Die bestimmung der mitleren räumlichen lichtintensität durch nur eine messung," Elektrotechn. Zeit. 29, pp. 595-597, 1900.

2. A. Roos, "Use of an integrating sphere in solar energy research," Solar Energy Materials and Solar Cells 30 , pp. $77-94,1993$

3. F. J. J. Clarke and J. A. Compton, "Correction methods for integrating-sphere measurement of hemispherical reflectance," Color Research and Application 11(4), pp. 253-262, 1986.

4. A. Roos, "Interpretation of integrating sphere signal output for nonideal transmitting samples," Applied Optics 30(4), pp. 468-474, 1991.

5. K. Grandin and A. Roos, "Evaluation of correction factors for transmittance measurements in single-beam integrating spheres," Solar Energy Materials and Solar Cells 33(25), pp. 6098-6104, 1994.

6. W. Platzer, "Solar transmission of transparent insulation material," Sol. Energy Mat. 16, pp. 275-283, 1987.

7. W. Platzer, "Directional-hemispherical solar transmittance data for plastic structure," Solar Energy 49, pp. 359-369, 1992.

8. D. Milburn and K. Hollands, "Solar transmittance measurements using an integrating sphere with broad area irradiation," Solar Energy 52, pp. 497-507, 1994. 